\title{
Patterns and Motivations of Code Switching and Code Mixing by Egyptian Facebook Users
}

\section{Safaa Abousoud Ahmed Abousoud}

\section{Abstract}

The Arabic Language is widely used all over the world. It has been influenced recently by certain factors. Two important constituents of these factors are Code-Switching (CS) and Code-Mixing (CM) from Arabic into other languages within the same discourse. The present study addresses the types and causes of CS and CM on face book. Furthermore, it handles the phenomenon of Franco-Arabic Code (FAC), which spreads rapidly via social media, especially facebook. FrancoArabic, as an apparent example of CS and CM, is a mixture of 'Araby' (the Egyptian word for Arabic) and "Inglizi" (the Egyptian word for English).The targets of this study focus on the socio-linguistic features of this phenomenon used by different generations via different websites and Facebook, in particular. It also tackles the types of CS and CM concerning their relation to computer-mediated communication.

The study designs and distributes a questionnaire to different categories of people in various work fields. It utilizes the qualitative and quantitative approaches, leading to the results. It depends on three variables, namely age, gender, and recent careers to compare the participants regarding the use of Franco Arabic Code. The sample of analysis covers 483 FB users. Moreover, the questionnaire comprises two types of questions; the first category is named YES/NO questions while the second one is open-ended questions to give the participants

(*) Demonstrator, Department of English, Faculty of Arts, Sohag University.

This paper is part of an M.A. thesis entitled : Patterns and Motivations of Code Switching and Code Mixing by Egyptian Facebook Users, Supervised by Prof. Bahaa el Deen M. Mazeed Faculty of Al- Alsun, Sohag University \& Dr. Thanaa Al Kady Faculty of Arts, Sohag University. 
more freedom to express their notions and motives. A set of studies handle the phenomenon of Franco-Arabic in different places and from different aspects. However, not a single study tackles it in terms of CS and CM, especially via FB, from the perspective of sociolinguistic CS depending on both qualitative and quantitative approaches.

\section{Introduction}

\subsection{Background of the study}

It is noticeable that there has been a widespread usage of social networking websites, especially Face Book (FB) causing a case of semi-addiction of such sites. Many categories of people utilize FB for many targets, including ensuring the idea of socializing, marketing for business, establishing wider relations, and sharing useful notions. However, it has harmful counterparts such as wasting time and useless pages.

The idea of switching between two languages exists heavily via $\mathrm{FB}$ based on the nature of certain situations, especially if a user or a group prefers to communicate using a specific language. FB has a coherent relationship to the concept of electronic discourse such as electronic emails, messages, and blogs. The massive prevalence of different computer-mediated genres including FB, Twitter, and WhatsApp has participated in the emergence of new youth styles of communication via the computer or cellphones.

Part of this study is dedicated to the category of youth because their "language is another important aspect related to non-standard orthographies" (Jaffe, Androutspoulos, Sebba, and Johnson, 2012, p. 319). They add that Chambers (1995) reported linguistic variation and change are especially apparent during adolescence.

\subsection{Context of the study}

The present study is a sociolinguistic study that aims at investigating the influence of the three variables of age, gender, and recent careers towards the phenomenon of FAC that is 
represented in switching between Arabic and English languages within the same situation.

(CS) is a broad term tackled by many scholars. It is defined as changing back and forth between two languages. This is largely obvious in website messages and pages. Some categories of participants adopt other languages rather than Arabic. Such categories will be limited through analyzing the questionnaire. For example, within a certain conversation, one participant may use an English word in the middle of the whole Arabic conversation. This kind of CS is largely used by various generations. It is supposed that by following these different patterns of $\mathrm{CS}$, they may have a distinguishable image. This may be justified by computers which were semi-restricted to the keyboards of Latin letters because the Arabic ones were seemingly absent.

The Arab users of communication services found themselves Facing hard problems. In other words, they have to focus on another language rather than Arabic. The most distinguishable criterion for this type of CS may be summarized in using numbers to compensate the Arabic letters which the foreign language does deal with. These letters seem to be strange in shape and content. Despite the young generation's knowledge of such letters and symbols, other categories of society, including parents, do not know the nature of the Franco-Arabic code. They have to learn such alien language, which has turned to be a code among different persons to enable them to know how their sons and daughters communicate with their friends.

The outline of the research is given as follows:

Section one is an introduction that covers the background, objectives, hypotheses, context, and significance of the study.

Section two shows the review of the relevant literature.

Part three discusses the theoretical background of the study. The study adopts Hoffman's (1991) model that addresses the types and motivations of CS and CM. 
Section four is dedicated to the tools and methods of the study which helped in collecting data.

Section five investigates the statistical analysis of the data depending on the responses of the participants to the questionnaire's items .

\subsection{Objectives of the study}

The main objective of this study is to investigate the reasons for using CS and CM on FB. It also highlights FrancoArabic Code (FAC) as an example of CS. Furthermore, it shows qualitatively why this code largely spreads among youth of different categories of participants. It indicates the most repetitive forms of $\mathrm{CS}$ and $\mathrm{CM}$ among the Egyptian $\mathrm{FB}$ users. It refers quantitatively to the percent of utilizing Franco-Arabic (FAC) among Egyptians.

\subsection{Research problem and questions}

This study investigates the classifications and reasons of CS and CM. Additionally; it seeks to answer the following questions as well as the questions of the questionnaire on which it is based.

1. What are the different kinds of CS and CM which are nearly common among Egyptian FB users in their messages?

2. Why do FB users tend to use FAC?

3. When do they use this code as a reflection of CS?

4. What are the reasons for using $\mathrm{CS}$ and $\mathrm{CM}$ in $\mathrm{FB}$ messages?

5. What are their justifications for using FAC?

6. Is the utilization of FAC confined to their friends, their relatives, or other categories? What are their reasons?

7. Does the usage of FAC depend on the specializations related to foreign languages? All the aforementioned open-ended questions are part of the main items of the study's questionnaire. The second part is yes/no or NEUTRAL questions, which include the following items:

1. Do you have any idea about FAC? 
2. Do you utilize it perfectly?

3. Is its usage justified to technological reasons?

4. Do you use it repeatedly on social media?

5. Does FAC threaten the Arab identity?

6. Does FAC possess cultural reasons?

7. Is FAC considered a developed style of language?

8. Is it restricted to youth only?

9. Do females tend to use FAC with emoticons more than males?

\subsection{Hypotheses of the study}

The present study tries to verify the following hypotheses:

1. Young people use FAC as a manner of showing progress, and civilization.

2. They utilize CS to show how they are cultured.

3. They, as young generations, handle this language as a secret code.

4. Youngsters in particular tend to utilize this code.

5. Franco's usage is justified by technological reasons.

6. Males use FAC less than females.

7. FAC has historical roots.

8. FAC has psychological and social reasons that enabled it to spread.

9. Using FAC correlates with identity.

10. FAC's occurrence is limited to social media?

\subsection{Significance of the study}

This study highlights the reasons for CS and CM. In addition, it identifies the most common types of CS and CM within the frame of FB. It is hoped that the study can cover the phenomenon of FAC which may influence the Arabic language. Although Arabic is not a poor language, as it has many aspects of rhetoric, many people do not appreciate its value. Furthermore, the study attempts to answer the questions raised qualitatively by analyzing certain samples of the study along with the quantitative analysis of the questionnaire's items. It verifies the aforementioned hypotheses. 


\section{Literature Review}

\subsection{The correlations of FB, technology, and FAC}

There is a coherent connection between the three aforementioned elements in which, they interact with each other. As Cameron and Panovic (2014) mentioned that people do not coin things with no practical purpose in mind. Technological achievement is always a reaction to already apparent needs or changes in the wider society. They reported that within the last 30 years, there were more dramatic developments with the production of texts, thanks to the knowledge of digital technology. They focused on the idea of social needs for every innovation. They argued that the Internet applications are interactive media, where participants are not mere passive recipients of content but they are able to interact. They assured that individuals do not follow a new practice not only because it is available technically, but also because of social Factors, relevance, usefulness, and pleasure.

Albirini (2016) argued that changes in the digital media affected many concepts and formulations in sociolinguistic research in which he referred to the model of Blommaert (2010) saying that globalization forces sociolinguistics to change its classic distinctions and biases and to rethink itself as a sociolinguistics of mobile resources, framed in terms of transcontextual network, and movements(Blommaert,2010.p.1). According to the technological changes, which appeared heavily on the Internet sites, new kinds of CS began to penetrate the sphere of social media on FB. Thus, Albirini (2016) gave a hint to (Jaffe et al., 2012; Dorlejn \& Nortier, 2009; Hinrichs, 2006) stating that the most remarkable multilingual activity online "is the use of more than one linguistic variety in the course of a single sentence or a piece of discourse in a way similar to CS in real-life situations" (p. 132). He added that the nature of online CS is mainly based on stylistic and social norms or patterns that are similar to those of spoken communication. 
Kobler et al (2010) reported that over 400 million active users prefer using FB as one of the largest social networks on the Internet and one of the most influential media in social interactions. They analyzed FB micro-blogging function that appears through users' status updates. They concluded that people began to addict to FB.

Liberman (2014) investigated the purposes of using FB.

These purposes include "keeping in contact with friends overseas, organizing meetings, social networking, distracting

myself, sharing pictures, planning events and parties, complaining about things, making politics matter, talking to classmates, uploading photos, fun activities, getting to know

people in Canterbury, and checking inbox" (p. 31).

Taha (2015) referred to the construction of FAC confirming that it consists of Arabic words which are written in English letters in addition to other symbols which are used to replace the missing letters in both English and French. He gave the example of صباح الخير to be in the form of 'saba7 215air' when transliterated in FAC. What happened in this phrase is that the Arabic letter $(\tau)$ is replaced with the digit $\{7\}$, and the Arabic letter $(\dot{\zeta})$ is replaced with the digit $\left\{7^{\prime}\right.$ or 5$\}$, (Taha, 2015) confirmed the definition of (Skiba, 1997; Abu Mathkour, 2004) concerning what is meant by CS to be the usage of two or more languages between participants in a conversation. Albirini (2016) focused on the idea of multilingualism in the universe of cyberspace communication.

Taha (2015 as cited by Albirini 2016) mentioned that multilingualism is represented in several shapes. He continued saying "one important facet of multilingualism is reflected in the designs, frames, advertisements, web page content, discussion forums, chat rooms, and e-mails, which are often encoded in a different language, but particularly English" (( taha,2015 as cited by Albirini, 2016, p. 263). These words can be applicable to the utilization of FAC on FB pages and comments as a shape of multilingualism in which English 
letters are written instead of the Arabic alphabet. Because FB users deal both English and Arabic, they are called bilinguals since they utilize two varieties within the same situation.

\subsection{Sociolinguistic features of code, emotions, and orthographic description}

The term "code" has been discussed by many studies including (Wardaugh, 1986, as cited in Cakrawati, 2012) to which code is defined as a language or system that two or more persons use to communicate. As a result, choosing a specific code is a situation in which groups of people communicate with others, selecting their appropriate style of language. Wardaugh's explanation concerning the real meaning of the code is applicable to the Egyptian youth's tendency to choose the FAC code to communicate on the virtual community of FB. The code also expresses the manner of dealing with something through a language, selecting a certain dialect, style, and register. Nilep (2006) referred to the words of Blom and Gumperz, arguing that "social incidents, participants, setting, and topic restrict the selection of linguistic variables" (Nilep, 2006, p. 7).

Cakrwati (2011) defined a code as "a system that is used by people to communicate with each other...It is a symbol of nationalism that is used by people to communicate in a particular language, register, or style on different occasions and for different purposes (Cakrawati, 2011, p.12). According to Mahootain (2006, as cited in Abusa'aleek, 2014, p. 511), Code Switching is "a linguistic phenomenon that is commonly occurring in bilingual and multilingual speech communities. It refers to the systematic use of two or more languages or varieties of the same language during oral or written discourses

Maizarevalo (2014) suggested that emotions have a deep relation with Computer-Mediated Communication (CMC). She referred to emotions' functions according to FB users. She linked the use of emotions and gender via FB. She figured out the reasons for using emotions as a substitute for visual 
characteristics of the real world. She pointed out that there are many differences between CMC and face-to-face (FTF) communications, represented in the channels utilized by both of them. For instance, FTF communication depends on different channels including auditory, gestural, and visual.

The variation used in the orthography of FAC by FB users is clearly noticed. To this point Jaffe et al. (2012) were convinced with the point that "where there is variation, there is in practice social meaning (p.32). They contradicted the logic of Milroy (1991) confirming that spelling is the most uniform and fixed level of language use. Jaffe et al. argued that the "orthographic practices are usually made in particular, social, historical, and cultural contexts" (2012, p.320).

There are many reasons for a group of people to use nonstandard orthography. It is indicated that to write in a nonstandard variety is a technique to influence and manipulate orthography (Themistocleous, 2010).

\section{Theoretical Framework}

\subsection{Introduction}

Code switching refers to changes of code (variety) by individuals. People sometimes switch code within a domain or social situation. Code switching is not arbitrary, but it has a necessary need.it refers to a choice made by the individual. There are social factors which are involved in code switching. According to Holmes (2001), these factors include participants, topic, affective functions, solidarity, shared ethnicity, social distance between participants (holmes, 2001, p.15). Code switching can reflect associations of certain topics with a language. Code switching can serve as a kind of euphemism or some kind of 'emotional buffer' of the unwanted taboo words. It is a very broad term that is not restricted on the area of speech, but it extended to include styles and techniques in treatment among participants.

These styles can change from being formal and informal or from being casual to be a professional. There are two teams 
of scholars who dealt with the phenomenon of code switching. The first team dealt with both code switching and code mixing interchangeably without any difference especially those who study syntax or morphology. On the other hand, the other team handles them differently. They perceive that code switching is used by individuals to show their proficiency in language in addition to multitude of languages they speak, but code mixing is used by people unintentionally simply because they do not know the correct word or phrase.

\subsubsection{Patterns of CS according to Hoffman's (1991) model}

a. Emblematic switching: This kind of switching includes the forms of tags and exclamation.

b. Inter-sentential CS: This kind happens for the entire clauses or sentences.

c. Formulating continuity with the previous speaker in order to ease the process of communication.

d. Intra-sentential switching: It occurs within a clause or sentence boundary.

\subsection{Types of CM according to Hoffman's (1991) model}

a. Intra-sentential CM: This type of CM exists at the level of the phrase and clause.

b. Intra-lexical CM: This type occurs within word boundary. For example, kuenjoy (English enjoy with the Swahili prefix ku-, meaning 'to').

c. Involving a change in the pronunciation: This kind of CM happens at the phonological level when some people pronounce a certain word of a specific phonological structure to modify it to their phonological structure. For example, in their writings on FB, users substitute the English letters "eat" with the digit '8' word in "gr8". In the word 'love u 2much', they substitute the pronunciation of 'you' with the letter ' $u$ ' and the word 'too' with the digit ' 2 '. 


\subsection{Reasons of CS and CM according to Hoffman's model}

Hoffman (1991) perceives that the participants utilize CS and

$\mathrm{CM}$ to achieve the following functions and motivations.

A. Quoting a person's words

Participants use CS or CM to quote a famous expression, proverb, or famous saying of prominent figures.

B. To express solidarity

A person who uses a language that is not his original one has a desire to be more serious or solid towards something. Thus, he/she voluntarily or involuntarily prefers to switch from the second language to the mother tongue or he/she can switch from the mother tongue to the second language.

C. Inserting sentence fillers or sentence connectors (interjection)

An interjection is words or expressions added to express surprise or strong emotions or to attract attention. It can be found in the shape of a short exclamation like hey! Well! Look...etc. They are more utilized in speaking than in writing.

D. Repetition used for clarification

If the bilingual person wants to add more clarification and certainty to the speech, he/she switches between codes and varieties.

E. Talking about a particular topic

Participants in certain situations choose to utilize different codes rather than their original ones. These codes are kept to express certain topics such as emotions, feelings, insults...etc. Cakrawati (2011) added more motivations of CS and CM:

1. Because of a real lexical need

The lack of lexical items in one language obliges its users to switch or mix elements that go with their need in a specific situation.

2. To exclude others from communication

When the interaction among participants is limited to a specific audience, they tend to use codes that are alien to other audience to preserve the specification of their topics. For instance, when 
certain users of FB comment on their FB pages or send messages, they use FAC to be restricted to their friends only.

3. To soften or strengthen a request

Sometimes, people tend to shelter to another variety away from their native one. This is accomplished to ask for something politely.

\subsection{Filling a gap in the literature}

The above-mentioned studies are not comprehensive in analyzing the e-discourse of FAC. They investigated the general reasons of adopting the phenomenon of FAC, but they did not focus on the private reasons that motivate the users to adopt such a phenomenon. The present study also tackles the textual features of this language and its relation to the patterns and motivations of CS and CM. In addition, the studies did not focus on the linguistic variables of gender, age, social class, and educational level of the participants and their relation to FAC. Attwa (2012) comments that "Arabizi is becoming a trend that cannot be overlooked, especially in the realm of CMC" (p.22).

\section{Methodology}

\section{1 data collection}

This study is conducted by designing a questionnaire that is distributed to a randomly selected sample of 483 participants. Then, the data are collected and analyzed statistically to get the results. The questionnaires are distributed to conclude the quantitative result of the research depending on the linguistic features of age, gender, and recent career. Some questionnaires are distributed online via e-mails and FB pages, while others are distributed manually. (Rosen, Chang, Erwin, Carrier, and Cheever, 2010, as cited in, Luddy, 2014) report that youngsters reflect bad influence in the formal writing on the Internet, while they feel appositive reflection in the informal writing.

For the purpose of collecting data that are connected with CMC, Herring (1996) reports that "some authors have devised sampling techniques for constructing representative corpora, 
some have used questionnaires as data- gathering instruments, some have relied on ethnographic observation of naturally occurring interactions, and some combined these with other methods" (p. 5).

\subsection{Ethical issues}

Certain issues shall be considered concerning the ethical issues of collecting data. Herring (1996) refers to "the dilemmas" or ethical points of CMC data, as follows:

It is ethical to collect while "lurking" (reading without contributing in an electronic forum? To the extent that a forum is open to the public, one can argue that this practice is not different from collecting data from by eavesdropping on a conversation in a place such as a restaurant or an airport [...]. Some consider all CMC works to be published in a written form. They perceive that quoting it without crediting the source is in violation of copyright [...] CMC research policies (p.5).

\subsection{Description of data}

The corpus of the study comprises one conversations selected from FB pages. This sample is analyzed according to Hoffman's (1991) model to limit the types of CS and CM. The last six messages are analyzed according to the components of speech situation of Biber's (1988) model. The extracted messages are chosen accurately to reflect the phenomena of CS and $\mathrm{CM}$ within written messages in FAC.

\subsection{Models/tools}

The present study follows the models mentioned in the theoretical framework: Hoffman (1991) and Biber (1988). The study adopted the model of Hoffman (1991) in which he gave a comprehensive categorization of CS and CM types within any discourse. It also utilizes the model of Biber (1988). The study handles the quantitative results of the questionnaire distributed to a random sample of the Egyptian males and females who write their messages or comments in FAC.

\subsection{Procedures}


The researcher distributed a questionnaire to a random sample of participants from different places in Egypt, such as Sohag, Hurghada, Cairo, Alexandria, Minia, and Saudi Arabia. The questionnaire was sent via e-mails or FB messenger, in addition to questionnaires that were delivered by hand. Then, the participants sent it back after filling it in. The participants were categorized according to the sociolinguistic variables of age, gender, and educational background.

\section{Findings}

\subsection{Results of the open-ended questions (interviews)}

These questions are asked in interviews with participants. They are left open to give them full freedom to answer. They are as follows:

1. Why do you switch codes in your conversations?

Answers:

a. Because of the inability of the Arabic language to be suitable in most of our repeated situations (35 participants, with a percentage of $7.2 \%$ )

b. To show knowledge of more than one language in addition to acquiring new cultures (120 participants, with a percentage of $24.8 \%$ )

c. Because of the overspread of the English language in various daily fields. This affects the involuntary transference of words and idioms in sentences (203 participants, with a percentage of $42 \%$ ).

d. To communicate with others in an easier manner within any conversations (50 participants, with a percentage of $10.3 \%$ )

e. To focus on something in a different language to avoid being understood by other characters (75 participants, with a percentage of $15.5 \%$ )

2. Why do you mix codes in uttering your words?

Answers:

a. Because it is needed in the lectures of my department and outside as it is recommended by my professors to apply 
more situations in English (45 participants, with a percentage of $9.3 \%$ ).

b. Because I cannot sometimes find the exact word in Arabic to express the exact meaning $I$ intend to say (56 participants, with a percentage of $11.5 \%$ ).

c. According to the temporal condition (55 participants, with a percentage of $11.8 \%$ ).

d. When the other participants in conversation is knowledgeable enough in the personal utterance (64 participants, with a percentage of $13.25 \%$ ).

e. The more words are peculiar, the more they attract the attention of others (93 participants, with a percentage of $19.2 \%)$.

f. To keep privacy in various situations (25 participants, with a percentage of $5 \%$ ).

g. Because of linguistic and social reasons including the knowledge of more than one language (55 participants, with a percentage of $11.3 \%$ ).

h. To achieve the target of consistency to one language rather than another (15 participants, with a percentage of $3.1 \%$ ).

i. Because with more than one language, the individual tend to connect with others mixing the native words with foreign ones, so it is natural to code mix between more than one language within the same situation (75 participants, with a percentage of $15.5 \%$ ).

3. Is the word "language" a suitable term to refer to FAC?

Answers:

a. It is very suitable because the participants use it to communicate on FB depending on letters and words. Therefore, it achieves the target of the language, i.e. delivering and understanding the message among the participants. It seems like the language of signs and body, which have indications for each sign, [287 participants, with a percentage of $59.4 \%$ ). 
b. It is not suitable because it mixes words, letters, fragments, and numbers. Thus, it can be titled as a mixed style of language (196 participants, with a percentage of 40.5\%)

4. Why do you use FAC on FB?

a. Because it is easier and saves more time than writing in pure Arabic or pure English. It is very simple for the participants who know well how to use it but a bit difficult for participants who are unaware of it (59 participants, $12.2 \%$ ).

b. Because the young generations tend to the Arabic language in certain situations. (30 participants, with a percentage of $6.2 \%$ )

c. To keep up with technology, as well as the social and cultural development even just to pretend that he/she possesses an extent of culture. (107 participants, 22.1\%)

d. Because it is close to the slang language and expresses everyday speech (57 participants, with a percentage of $11.8 \%$ )

e. It is used in certain conditions when it is necessary (10 participants, with a percentage of $2 \%$ )

f. Because it spreads rapidly on FB in addition to its title as the language of social media (59 participants, 12.2\%)

g. because it is like a code among its utilizers (25 participants/ with a percentage of $5.1 \%$ )

h. To keep with the speech situation on FB and to avoid the perception of being inferior among friends because of the lack of realizing this language (19 participants, 3.9\%)

i. I sometimes use FAC in uttering taboo or impolite words without being understood by other users of FB (9 participants, $1.8 \%$ )

j. Because there are certain participants on FB from various places who do not understand the Arabic language, it is sometimes necessary to use FAC with them. (17 participants, $3.5 \%)$

$\mathrm{k}$. To avoid the wrong spellings and grammatical restrictions of Arabic and English. (13participants, 2.6\%) 
1. Because of the negative impact of English on Arabic (50 participants, $10.3 \%$ )

$\mathrm{m}$. For political reasons, it is possible to mix languages to speak in political issues without being understood except by those who know the code. (28 participants, with a percentage of $5.7 \%$ )

5. Is the utilization of FAC limited to your friends, relatives, or other categories? Why?

a. It is limited to my friends because they are the most familiar users of this language in addition to lacking the knowledge whether strange users know this kind of writing or not. To avoid embarrassing, it is advisable to use it with familiar users. (219participants, 45.3\%)

b. It is limited to my relatives because I am accustomed to chat with them in FAC. (30 participants, 6.2\%)

c. The degree of closeness or farness from others does not affect the use of this language because any person can use it if familiar (187 participants, 38.7\%)

6. Does the usage of FAC depend on the specializations related to foreign languages?

a. It differs from one person to another according to specialization because those who can communicate in a foreign language tend to apply FAC. (297 participants, 61.4\%)

b. It does not vary depending on the specialization because anyone can use it. (186 participants, with a percentage of $20.4 \%$ )

c. It depends on the situation itself ( 87 participants, with a percentage of $18 \%$ )

7. How will you behave if a formal body prevented the use of FAC? Why?

a. This will be a good decision because it is a difficult style of writing to be learned quickly AND to avoid its bad effect on Arabic (301 participants, with a percentage of 62.3\%)

b. A wrong decision because its usage is a personal freedom. It is not a formal language in interacting with others. 
This action will lead to a case of disorder among youngsters who are accustomed to write with this language. (147 participants, $30.4 \%$ )

c. I do not care because it does not represent a crucial matter for me. (35 participants, with a percentage of $7.2 \%$ )

8. To what extent can FAC affect Arabic? Give examples.

a. Moderate (97 participants, 20\%)

b. Low (65 participants, $13.4 \%$ )

c. High (329 participants, $68.1 \%$ )

\section{2 conclusions}

The present study investigated the patterns of CS and CM utilized by the Egyptians through the application of FAC. It results in answering and commenting on the questions of the questionnaire. It is concludes from the recent study that code switching is used less than code mixing, because participants tend to violate the stable standards of language to use a simplified code for their dealings with each other. The rest of the results are mentioned earlier in the previous section.

\section{References}

1. Abusa'aleek,A.O.(2014).A review of emerging technologies :Mobile assisted language learning (MALL).Asian journal of education and e-learning(ISSN:2321-2454,2(06).

2. Albirini, A. (2016). Modern Arabic sociolinguistics: Diglossia, variation, code switching, attitudes and identity. New York: Routledge.

3. Blommaert,J.(2010) .The sociolinguistics of globalization. Cambridge university press.

4. Attwa, M. (2012). Arabizi: A writing variety worth learning. The American University in Cairo, School of Humanities and Social Science.

5. Biber, D. (1988). Variation across speech and writing. Cambridge: Cambridge University Press. 
6. Cakrawarti, D. A. (2011). Analysis of code switching and code mixing in the teenlit Canting Cantiq by Dyan Nuranindya (Ph.D. dissertation). University of Diponegoro, Indonesia.

7. Cameron, D. \& Panovic, I. (2014). Working with written discourse. New York: Sage Publishing.

8. Chambers, Jack K. 1995. Sociolinguistic Theory. Oxford: Blackwell.

9. Dorleijn, M. (2016). Introduction: Using multilingual written Internet data in code-switching and language contact research. Journal of Language Contact, 9(1), 5-22.

10. Hoffman, C. (1991). An introduction to bilingualism. London: Longman.

11.Herring, S. (1996). Computer-mediated communication: Linguistic, social, and cross-cultural perspectives. Amsterdam: John Benjamins Publishing.

12. Mahootian 2006 code switching in K.brown(ed.),Encyclopedia of language and linguistics( $2^{\text {nd }}$. ed.).(vol2, pp.511-526)oxford:Elsevier.

13.MAÍZARÉVALO, C. (2015). A pragmatic and multimodal analysis of emoticons and gender in social networks. New Insights into Gendered Discursive Practices: Language, Gender and Identity Construction, 10.

14.Saville-Troike, M., \& Kleifgen, J. A. (1986). Scripts for school: Cross-cultural communication in elementary classrooms. Text-Interdisciplinary Journal for the Study of Discourse, 6(2), 207-222.

15.Jaffe, A., Androutspoulos, J., Sebba, M., \& Johnson, S. (Eds.). (2012). Orthography as social action: Scripts, spelling, identity and power (Vol.3). Berlin: Walter de Gruyter. (1-15).

16.Köbler, F., Reidl, C., Vetter, C., Leimeister, J. \&Krcmar, H. (2010). Social connections on Facebook: An explorative 
study on status message usage. Paper presented at $16^{\text {th }}$ Americas Conference on Information Systems, Peru. Available at SSRN: https://ssrn.com/abstract=1641178.

17.Liberman, S. (2014). Using Facebook as interactive learning environment in European political studies. European Political Sciences, 13(1), 23-31.

18.Maiz-Arevalo, C. A. (2014). A pragmatic and multimodal analysis of emoticons and gender in social networks. Academic.edu: Cambridge university press. [it is a paper in a book]

19.Milroy,L., Li, W., \& Moffatt, S. (1991). Discourse patterns and fieldwork strategies in urban settings: Some methodological problems for researchers in Bilingual communities. Journal of Multilingual \&Multicultural Development, 12(4), 287-300.

20.Nilep, C. (2006). "Code switching in Sociocultural linguistics." Colorado Research in Linguistics, 19(1), (1$11)$.

21.Themistocleous, C. (2010). Writing in a non-standard Greek variety: Romanized Cypriot Greek in online chat. Writing systems research, 2(2), 155-168.

22.Taha, M. (2015). Arabizi: Is code switching a threat to the Arabic language? Paper presented at the Asian Conference of Arts \& Humanities, American University of Sharjah, UAE. 